\title{
Fatores que afetam o reuso de objetos de aprendizagem
}

\author{
Liane Margarida Rockenbach Tarouco - Pós-Graduação em Informática na Educação \\ Claraluz Camargo Gris da Silva - CINTED/UFRGS \\ Anita Grando CINTED/UFRGS
}

\begin{abstract}
This paper presents and discuss factor impacting reuse of learning objects. Metadata, granularity and the authoring tool eXelearning, that provides support for reuse, are analyzed in terms of facilities for integrate and reuse learning objects.

\section{Resumo}

Neste artigo são apresentados e discutidos fatores que afetam a possibilidade de reuso de objetos de aprendizagem. São analisados e comentadas a questão dos metadados, a granularidade e as funcionalidades da ferramenta de autoria eXelearning que possui facilidades para integrar e reusar objetos de aprendizagem.
\end{abstract}

Palavras-chave: objeto de aprendizagem, reuso, eXelearning

\section{Introdução}

Os objetos de aprendizagem ganharam ampla aceitação e importância no contexto do desenvolvimento de material educacional digital em função de seu potencial de reusabilidade. Muitos benefícios são esperados da abordagem de reusabilidade tais como economia e eficiência do tempo para o desenvolvimento de recursos, além de melhorias nas estratégias de ensino aprendizagem. Todavia o custo, medido em tempo e esforço necessários para criar um objeto de aprendizagem, mostrou-se muito superior ao inicialmente previsto (Gonzales-Barbone e Anido-Rifon 2008). Estes autores também destacam que um exame do conteúdo criado aponta a existência de muita redundância, destacando que quando um mesmo conteúdo precisa ser trabalhado em diferentes situações de aprendizagem, usualmente a reutilização não é na intensidade que a adoção de objetos de aprendizagem deveria ensejar. A expectativa derivada da adoção da estruturação do conteúdo educacional digital, seguindo o modelo de objetos de aprendizagem, era de que haveria redução de custos em função do reuso de objetos de aprendizagem na composição de unidades de aprendizagem com agregação e/ou sequenciação de objetos de aprendizagem. Este processo permitiria compor com maior facilidade novas unidades de aprendizagem, contextualizadas tendo em vista diferentes públicos alvo e buscando como meta alcançar objetivos educacionais específicos.

Criar um objeto de aprendizagem reusável não é tarefa trivial. Dentre as dificuldades enfrentadas pelos desenvolvedores de conteúdo constata-se falta de recursos préexistentes e que sejam utilizáveis. Conforme salientado por Wiley (2009) a metáfora do objeto de aprendizagem como peça Lego, tende a limitar o escopo das opções de reusabilidade, pressupondo que implicaria apenas na remontagem de peças, tal como são encontradas. Mas a demanda, em termos de reuso, vai além desta mera montagem de peças prontas envolvendo o que este autor denominou de "4 Rs":

- Reuso - fazer e reutilizar cópias do material tal como se encontra

- Revisão - alterar ou transformar o material para que ele melhor se ajuste às necessidades educacionais em um novo contexto 
- Remixagem - mixar (combinar) o material para ajustar a necessidades educacionais diferentes

- Redistribuição - compartilhar o trabalho na íntegra, revisado ou remixado

Estas atividades seriam usualmente inibidas pelas leis de copyright que imperavam no cenário da publicação impressa desde há muito tempo. Mas surgiram no contexto da produção de conteúdo educacional digital novas licenças associáveis a conteúdos digitais, tais como as licenças Creative Commons, GNU ou Open Publication Licences. Estas licenças ensejam um reuso mais amplo, nos termos dos 4Rs sugeridos por Wiley o qual participa de um movimento amplo denominado Open Content (conteúdo aberto) que visa ensejar a reusabilidade de conteúdo educacional digital nos termos previstos nos 4 Rs e de forma análoga ao que ocorre com o movimento de software livre.

Mas, apesar deste novo cenário de conteúdo aberto, a taxa de reuso de objetos de aprendizagem não é tão elevada quando seria desejável. Um estudo realizado por Ochoa e Duval (2008) constatou uma taxa de reuso em torno de $20 \%$ no levantamento realizado pelos autores em um conjunto limitado de repositórios. Também do Brasil percebe-se que o reuso de objetos de aprendizagem não é tão intenso quanto seria desejável. A falta de orientação tanto para a produção e armazenamento de objetos de aprendizagem reusáveis, como para seu reuso são elementos críticos para um maior reuso, tal como destaca Alsubaie (2009).

É preciso investigar os fatores que impactam o reuso de objetos de aprendizagem e este é o escopo inicial do presente artigo. Inicialmente serão discutidas as questões inerentes ao uso de metadados para dar melhor visibilidade aos objetos de aprendizagem. A seguir aspectos derivados do tamanho dos objetos de aprendizagem e seu impacto no reuso serão comentados. Adicionalmente serão apresentadas algumas estratégias e ferramentas que podem ajudar a aumentar o reuso de objetos de aprendizagem.

\subsection{Metadados para promover o reuso}

Uma das hipóteses mais frequentemente encontradas na bibliografia relacionada ao reuso de objetos de aprendizagem é a de que o uso de metadados para descrever os objetos de aprendizagem facilita sua recuperação. A ampla disseminação do padrão IEEE 1482 - Learning Object Metadata, bem como o uso de outros padrões mais simples, tal como o Dublin Core, constituíram uma decorrência desta crença. Naturalmente esta hipótese está associada com o armazenamento dos metadados e possivelmente dos próprios objetos de aprendizagem em um repositório.

No primeiro caso, quando são armazenados apenas os metadados, alguns autores designam este tipo de sistema como referatório e ele contém apenas a referência ao objeto de aprendizagem em si. Um metadado previsto contém o URL onde o objeto está disponível. Esta solução tem a seu favor o fato de que os autores podem mais facilmente manter a gestão dos objetos de aprendizagem, efetuando ajustes ou aprimoramentos nos mesmos. Todavia, por vezes, ocorre a mudança do local de armazenamento do objeto de aprendizagem sem que o correspondente metadado seja atualizado e isto leva à existência de registros no referatório que não contém mais o endereço correto e atualizado do objeto de aprendizagem e portanto aquele objeto tornase menos acessível pois somente os que conhecem seu novo endereço poderão encontrálo. 
No segundo caso, onde o repositório contém os metadados e o próprio objeto de aprendizagem tem-se como vantagem evitar esta perda de acesso mas os repositórios são usualmente planejados para que a cada registro de objeto de aprendizagem corresponda um arquivo que usualmente é uma versão compactada do conjunto de arquivos que podem estar incluídos em um objeto de aprendizagem. Se o objeto tiver que existir em mais de um formato, SCORM, IMS, página web etc... torna-se necessário buscar uma solução para acomodar estas diferentes versões de um mesmo objeto.

Em essência, cabe salientar que o uso de metadados associados aos objetos de aprendizagem facilita o processo de busca e recuperação de objetos adequados para os fins pretendidos pois, conforme destaca Billings (2010) é preciso avaliar se e como os OAs existem poderão ser usados tendo em vista os objetivos educacionais. Usualmente a análise de reuso de objetos de aprendizagem fica bastante centrada em aspectos tecnológicos mas, conforme destaca Gkatzidou (2010) estes aspectos não podem sobrepujar-se aos aspectos pedagógicos. Os metadados permitem refinar a busca filtrando não só por palavra chave mas também pelos atributos inerentes a qualquer um dos metadados. No caso de utilização dos metadados previsto no padrão LOM (IEEE 2002) a categoria educacional oferece um importante elenco de informações para o professor autor avaliar se determinado objeto de aprendizagem é apropriado ou não. No projeto CESTA (2003), por exemplo, foram utilizados os seguintes atributos da categoria educacional:

- Tipo de interatividade - modo predominante de aprendizagem (ativa, expositiva, mista)

- Recurso de aprendizagem: tipo específico do objeto (exercício, simulação, questionário, diagrama, figura, gráfico, índice, slide, tabela, teste, experiência, texto, problema, auto-avaliação, palestra)

- Nível de interatividade - grau de interatividade (muito baixo, baixo, médio, alto, muito alto)

- Usuário final esperado - tipo de usuário para o qual foi desenvolvido o objeto (professor, autor, aluno, gerenciador)

- Ambiente de utilização - escola, faculdade, treinamento, outro

- Faixa etária - idade do usuário final esperado

- Descrição - comentários sobre como esse objeto deve ser usado.

Mas apenas o uso de metadados não assegura o apropriado reuso dos objetos de aprendizagem depositados nos repositórios. A granularidade dos objetos e aspectos de seu projeto podem afetar seu reuso tal como será comentado nas seções seguintes.

\subsection{A granularidade e o reuso}

Wiley refere o trabalho de Reigeluth e Nelson de 1997 (apud Wiley 2002) que sugerem que quando os professores começam a trabalhar com algum material educacional, muitas vezes dividem/segmentam o material e remontam as partes de modo a atender seus objetivos educacionais específicos. A proposta dos objetos de aprendizagem viriam facilitar o trabalho dos professores ensejando a produção de materiais educacionais digitais já decompostos e mais apropriados para serem reusados.

Assim, o grau de granularidade de um objeto de aprendizagem é um aspecto costumeiramente ligado à possibilidade de reusabilidade. É senso comum que quanto 
menor a granularidade maior a reusabilidade. Em função disto, OAs de menor tamanho e com menos conteúdo teriam maior possibilidade de reuso do que OAs com conteúdos mais extensos, ou com pouca possibilidade de decomposição. Mas outros fatores também são importantes para que um objeto de aprendizagem seja reusável, conforme destaca Sanz-Rodriguez (2010):

- Auto-contido - deve fazer sentido por si mesmo. Referências a outros objetos diminuem a reusabilidade

- Modular - deve poder ser facilmente combinado com outros para formar estruturas compostas

- Granularidade apropriada ou passível de decomposição

- Rastreável - deve pode ser identificável e encontrado via metadados

- Modificável - possibilidade de reformular para um uso diferente do que foi originalmente proposto

- Usável - deve ser fácil de usar e deve ter uma interface intuitiva

- Padronizado - atender a algum padrão ou especificação (cores, letras, navegação, interface etc...)

A proposta de Sanz-Rodrigues (2011) para avaliar a reusabilidade de um objeto de aprendizagem também utiliza como métrica conceitos de coesão, acoplamento, tamanho e complexidade e portabilidade.

O presente trabalho busca identificar uma ferramenta que facilite o reuso de objetos de aprendizagem com vistas a atender a estes critérios pois, apesar da consolidação da importância da reusabilidade ela não se configura atualmente como uma prática disseminada. Isto ocorre em função de dificuldades práticas que os educadores/autores encontram na autoria e montagem de objetos de aprendizagem. Adicionamente, atitudes sócio culturais existentes, relativas a formas de colaboração e compartilhamento, têm sido também identificadas como barreiras potenciais ao reuso, tal como comentado por Elliot (2008).

\subsection{Suporte para reuso}

Uma forma de tornar a reusabilidade mais recorrente é mediante o uso de ferramentas de fácil manuseio que possibilitem a composição de novos OAs, integrando OAs existentes nos termos dos 4 Rs sugeridos por Wiley, assim como a utilização de padrões de empacotamento que abranjam diversas plataformas. Adicionalmente é preciso que sejam atendidos os quesitos de reusabilidade sugeridos por Sanz-Rodriguez (2011).

A composição de novos objetos de aprendizagem mediante simples agregação de objetos existentes, ou com sua revisão e/ou remixagem demanda uma ferramenta integradora que facilite estas operações por parte dos educadores mesmo para os que não tenham fluência digital.

O quarto R (Redistribuição) demanda uma solução que facilite o uso do novo objeto em diferentes ambientes. Neste caso, a utilização de padrões de empacotamento, tal como SCORM ou IMS, é uma estratégia recomendada para facilitar a redistribuição. 
As autoras tem trabalhado com uma ferramentas de autoria que oferece interessantes alternativas para apoiar a integração de Objetos de Aprendizagem nos termos dos 4Rs propostos por Wiley. Trata-se do software eXe Learning que será comentado nas seções seguintes.

\section{Uma ferramentas integradora de OAs}

$\mathrm{O}$ eXe - elearning XTML editor (eXe) é uma ferramenta livre e de código aberto criada para facilitar a autoria de OA's. Ela oferece uma série de recursos para criar e configurar componentes educacionais para o objeto de aprendizagem. Estes recursos são denominados no âmbito desta ferramenta, iDevices (dispositivos instrucionais) e permitem ao autor incorporar (Reuso), ajustar (Revisar), combinar (Remixar) conteúdo educacional digital de acordo com a sua intenção ou planejamento pedagógico.

A ferramenta eXe dispõe de diversas funcionalidades básicas inerentes a cada um dos iDevices, que permitem integrar OAs primitivos como imagens, textos, áudios, vídeos e pequenos testes construídos pela própria ferramenta, além de possibilitar a edição de texto a ser usado como moldura para os OAs incluídos, com vistas à contextualizar a unidade de aprendizagem para a situação de aprendizagem pretendida pelo professor/autor. A figura 1 mostra a visão geral da ferramenta e na janela inferior à esquerda a relação dos iDevices básicos que são disponibilizados a partir da instalação do software. Uma detalhada descrição de cada um dos iDevices pode ser encontrada no site do software (http://exelearning.org).

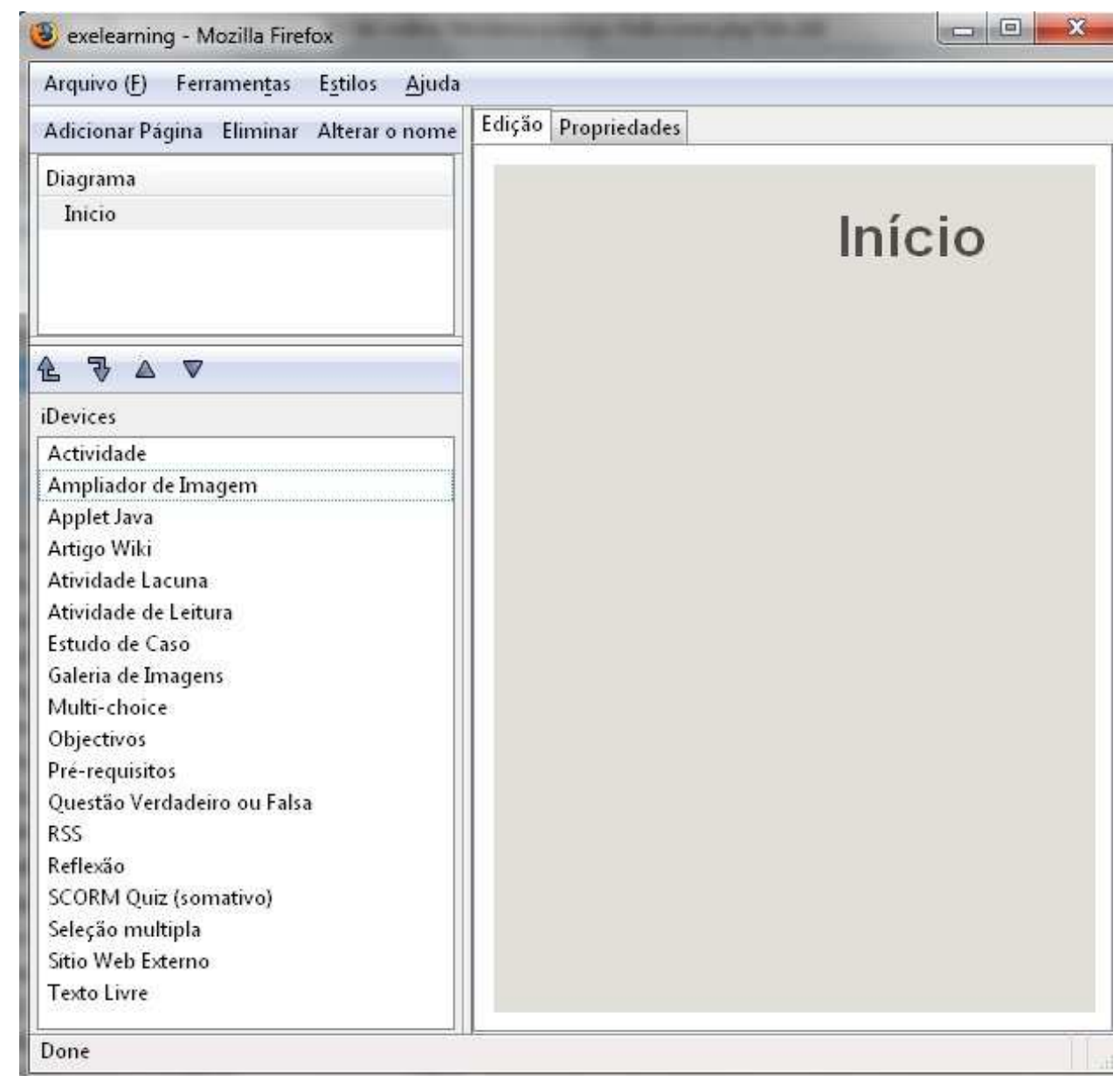

Figura 1: eXe e seus iDevices 
É possível criar novos dispositivos instrucionais, a partir do editor de iDevices presente na aba Ferramentas. Para salvar o material sendo elaborado, esta ferramenta de autoria produz um arquivo com extensão .elp (elearning packet) que poderá ser transportado para outros computadores e lá continuar a ser trabalhado.

Uma vez que o objeto de aprendizagem esteja pronto, o resultado pode ser exportado para diferentes formatos, tal como ilustrado na figura 2. Neste formato o objeto de aprendizagem é preparado para ser incluído em sites web, em ambientes virtuais de aprendizagem ou mesmo em algum outro meio de disseminação tal como CDROM ou pendrive.

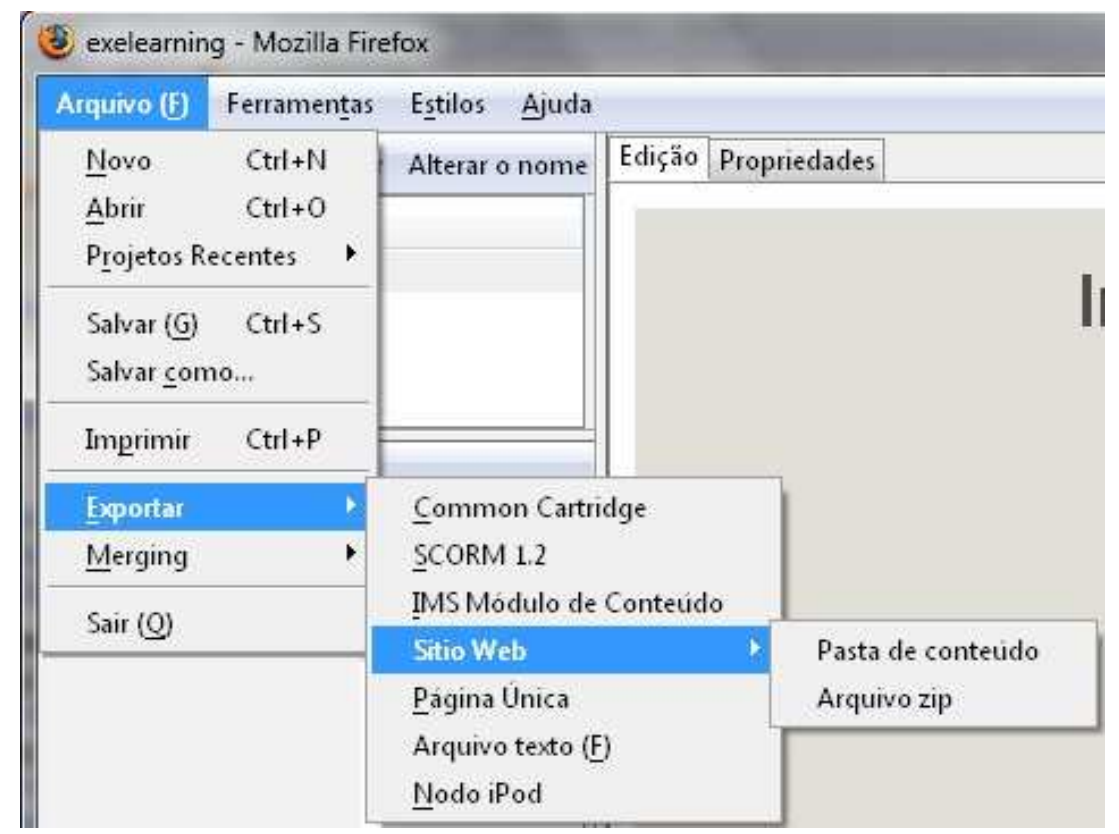

Figura 2: Opções de exportação de dados no eXe

As opções para exportar o objeto incluem formatos mais simples tal como um conjunto de arquivos de texto ou páginas organizadas em um diretório ou pasta ou mesmo compactados em um arquivo do tipo zip. Incluem também formatos, como o SCORM, que geram pacotes prontos para serem transferidos para ambientes virtuais de aprendizagem e que podem conter atividades de verificação de aprendizagem cujos escores serão considerados pelo AVA e atualizam o registro de notas do aluno que utilizar este objeto de aprendizagem. Para dar suporte a dispositivos móveis existe o formato de exportação "Nodo iPod" que gera arquivos compatíveis com os dispositivos portáteis da Apple.

\subsection{Integração de OAs criados com eXe}

$\mathrm{O}$ eXe facilita a construção de unidades de aprendizagem que integrem OAs previamente criados com o próprio software de autoria. Conforme referido na seção anterior, as unidades de aprendizagem (pacotes de conteúdo) construídas através do eXe são armazenados em arquivos com a extensão .elp (elearning packets). Para integrar um pacote previamente desenvolvido em outro, em construção, o autor deve selecionar no menu superior primário do eXe as opções: Arquivo>Merging>Inserir Pacote, tal 
como ilustrado na figura 3; na janela que abrirá será possível selecionar o arquivo .elp que o autor deseja unir ao pacote atual e aquele novo pacote será integrado ao projeto.

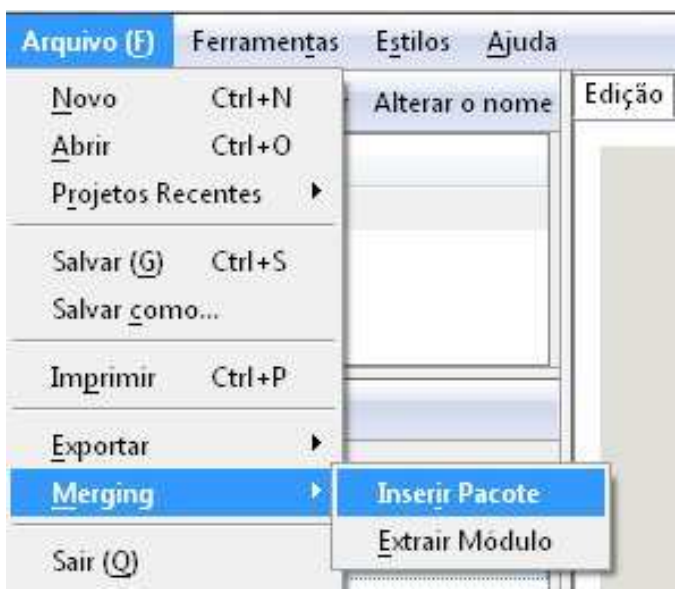

Figura 3: Inclusão de pacotes prontos

Também é possível extrair módulos de um arquivo .elp e transformá-los em novos arquivos .elp. Essa opção está disponível em: Arquivo>Merging>Extrair Módulo (também ilustrada na figura 1). Isso possibilita que se (re)utilize fragmentos da estrutura de um OA, tal como Reigeluth e Nelson de 1997 (apud Wiley 2002) constataram que os professores costumavam fazer.

\subsection{Inclusão de conteúdo gerado por outras ferramentas de autoria}

A ferramenta integradora eXe oferece uma gama de iDevices mas embora este conjunto contenha algumas opções para criar atividades interativas, elas não são tão amplas como as existentes em outras ferramentas como o Hotpotatoes ou mesmo tão flexíveis como o que se pode criar com uma linguagem de programação tal como Java.

Os iDevices para ensejar interatividade disponíveis na ferramenta eXe permitem criar exercícios de escolha simples, completar lacunas e questões do tipo falso-verdadeiro. Os professores autores de conteúdo educacional digital conhecem e apreciam usar também outras opções em termos de exercícios que oportunizem a interatividade e respostas ativas por parte dos alunos bem como uma auto-avaliação básica. Ferramentas como o Hotpotatoes (2011) ensejam criar, além das questões como as da ferramenta eXe, a outros tipos de exercícios, incluindo frases embaralhadas, questões de associação e palavras cruzadas.

Com a ferramenta integradora eXe pode-se importar os exercícios criados com esta e outras ferramentas de autoria. No caso de Hotpotatoes, existem diversas formas para incluir um objeto de aprendizagem produzido com esta ferramenta. Uma das estratégias consiste simplesmente em usar o iDevice Web Externo e ali incluir o endereço da página HTML com o exercício desenvolvido com o Hotpotatoes. A ferramenta de autoria eXe inclui, automaticamente, no pacote elp sendo construído, não apenas a página indicada mas também as imagens que ela porventura contenha. Assim o objeto de aprendizagem incluído desta maneira passa a integrar o pacote elp e poderá ser portado para outros ambiente para ser também reusado. Mas cabe destacar que, como apenas a página HTML produzida com o Hotpotatoes é incluída e não os arquivos editáveis com os quais o Hotpotatoes trabalha e que podem ser sucessivamente editados, 
o objeto de aprendizagem gerado com o Hotpotatoes e incluído no pacote elp não será facilmente editado. Por facilmente editado subentende-se a possibilidade de editar usando a ferramenta de autoria Hotpotatoes. Um usuário mais experiente sempre poderá editar a página HTML diretamente alterando o código Javascript nela contido ou os comandos HTML e textos associados. Mas isto não é o procedimento usual. Portanto, para preservar a reusabilidade dos objetos de aprendizagem criados com Hotpotatoes, os mesmo deveriam ser armazenados em repositórios de objetos de aprendizagem tanto em formato final (HTML) como no formato editável, próprio de cada ferramenta. Esta estratégia também deve ser usada para os objetos criados com a ferramenta eXe. Para que possam ser reusados, revisados e remixados é preciso que os objetos de aprendizagem reusáveis sejam armazenados em repositórios de objetos de aprendizagem tanto no formato final, em uma ou mais dentre as formas de exportação possibilitadas pela ferramenta, como também no formato editável (elp).

Objetos de aprendizagem criados com Hotpotatoes também poderão ser incluídos no pacote elp mediante inclusão do código fonte HTML da página com o exercício em uma janela apresentada pelo iDevice Applet Java. Este iDevice permite incluir qualquer tipo de Applets Java o que abre uma opção de inclusão de uma grande quantidade de objetos de aprendizagem atualmente existentes para simular dispositivos ou fenômenos físicos, além de opções com ferramenta de autoria externa como o Geogebra tal como descrito em Mussoi (2011). Com este iDevice do eXe, pode-se agregar muitos tipos de arquivos ao objeto de aprendizagem sendo produzido. Os Applets Java são programas desenvolvidos em linguagem Java, podendo ser inseridos dentro de códigos HTML. Existem diversos tipos de aplicativos desta espécie como calculadoras, jogos e outros aplicativos disponíveis livremente para download na internet. O modo de inserção desse tipo de material em um projeto do eXe é semelhante ao processo de inclusão de sítios externos, sendo que o iDevice Applet Java possibilita que se adicione o código fonte desses programas.

Com estes exemplos pretendeu-se evidenciar os recursos que são encontrados em uma ferramenta de integração que enseja a criação de objetos de aprendizagem usando amplamente as opções dos 4Rs. Existem outras ferramentas integradoras que também permitem trabalhar com eficiência dentro de uma abordagem de reuso de objetos de aprendizagem e é importante que seu uso seja disseminado pois elas tem potencial para alavancar o reuso de objetos de aprendizagem catalisando assim a produção de mais objetos de aprendizagem que por sua vez poderão ser reusados e todo um efeito de cascata pode resultar em mais e melhor conteúdo educacional digital.

As unidades de aprendizagem produzidas com a ferramenta eXe são de fácil reorganização e constituem ferramentas poderosas e de fácil manuseio para a produção de objetos de aprendizagem. Ela enseja também a possibilidade de trabalho colaborativo, com equipes trabalhando simultaneamente em segmentos do objeto de aprendizagem que podem ser posteriormente integrados mediante simples importação de arquivos elp.

\subsection{Exportar para portabilidade}

Adicionalmente a ferramenta de autoria eXe permite ao autor dar um acabamento ao seu trabalho com vistas a facilitar a portabilidade do objeto de aprendizagem para diferentes ambientes onde poderá ser usado pois a ferramenta permite a exportação do 
resultado do trabalho de autoria para formatos tais como: IMS Content Package, Common Cartridge, SCORM 1.2, pasta de conteúdo com o conjunto de arquivos no formato de páginas web ou pasta de conteúdo no formato zip, simples página web autocontida, no formato txt e no formato de notas para iPod. Essa variedade garante atingir uma gama de AVAs, sendo os formatos IMS e SCORM os mais usuais e compatíveis em ambientes virtuais de aprendizagem como o Moodle.

O resultado deste processo pode ser disponibilizado em Repositórios de Objetos de Aprendizagem tais como BIOE, CESTA, MERLOT, LABVIRT/USP entre outros. Os objetos depositados nestes repositórios podem então ser recuperados, a partir de busca baseada nos metadados associados a cada um e serem então transferidos para uso em AVAs (Ambientes Virtuais de Aprendizagem).

É importante ressaltar que a ferramenta eXe também tem a possibilidade de que o autor especifique metadados a serem associados com o objeto em produção. Embora o conjunto de metadados contemplados não seja o mesmo previsto em repositórios nos quais o objeto venha a ser depositado, a ferramenta permite trabalhar com um subconjunto relevante de metadados que podem ser aproveitados no processo de catalogação do OA no repositório. No caso dos objetos de aprendizagem exportados em formato SCORM as interações do estudante com o material podem ser monitoradas, acompanhadas e registradas no ambiente (Tarouco, Dutra, Ávila e Grando, 2007). Esses fatores favorecem uma avaliação e acompanhamento detalhado do processo de aprendizagem do aluno por parte do professor/instrutor, fornecendo dados para um rastreamento ou monitoramento das interações com os materiais disponibilizados, assim como possibilita ao aluno ter acesso a esses registros garantindo que ele acompanhe seu próprio progresso. O empacotamento de OAs em formato SCORM também garante ampla portabilidade em diferentes ambientes virtuais de aprendizagem, pois este formato tem sido cada vez mais adotado por diversos AVAs.

\section{Conclusões}

$\mathrm{O}$ artigo apresenta algumas estratégias para reusar OAs as quais podem alavancar a produtividade do professor autor de OA. Os aspectos ressaltados na presente análise permitem concluir que a utilização da ferramenta eXe Learning aliada a uma percepção ampliada das possibilidades que podem ser alcançadas com a reusabilidade de OA`s pode possibilitar o alavancamento da produtividade do professor autor desses materiais, na medida que este passa a construir, reusar, revisar, remixar e redistribuir esses objetos seguindo estratégias que proporcionem dinamizar tanto o seu processo de autoria, como o de outros que virem a utilizar os objetos por ele produzidos.

Assim dentre os fatores que impactam o reuso de objetos de aprendizagem foi ressaltado neste trabalho tanto a importância da promoção de uma filosofia de conteúdo aberto que permita o reuso nos termos dos 4Rs como a existência de ferramentas apropriadas para o trabalho de revisar e remixar. A análise da ferramenta de autoria eXe permitiu constatar que a esta ferramenta tem potencial para apoiar a produção de objetos de aprendizagem reusáveis que atendam aos quesitos sugeridos por SanzRodrigues (2011) de coesão, acoplamento, tamanho e complexidade e portabilidade bem como para reusar objetos de aprendizagem.

\section{Referências}

V. $9 \mathrm{~N}^{\mathrm{o}} 1$, julho, 2011 
- ALSUBAIE, M.; ALSHAWI, M. Reusable objects: Learning object creation cycle. Proceedings - International Conference on Developments in eSystems Engineering, DeSE 2009, pp. 321-325.

- BILLINGS, D.M. Using reusable learning objects. Journal of Continuing Education in Nursing, 41 (2), 2010, pp. 54-55.

- CAMERON, T.; BENNETT, S.. Learning objects in practice: The integration of reusable learning objects in primary education . British Journal of Educational Technology, 41 (6), 2010. pp. 897-908.

- CESTA. Coletânea de Entidades de Suporte ao uso de Tecnologia na Aprendizagem. 2003 Disponível em http://www.cinted.ufrgs.br/CESTA/ Acessado em 10 de Maio de 2011.

- ELLIOT, Kristine. SWEENEY, Kevin. Quantifying the reuse of learning objects. Australasian Journal of Educational Technology 2008, 24(2), 137-142

- eXeLearning . Disponível em http://exelearning.org/wiki. Acessado em 20 de março de 2011

- GKATZIDOU, V.; PEARSON, E. Exploring the development of adaptable learning objects. A practical approach. 10th IEEE International Conference on Advanced Learning Technologies, ICALT 2010, art. no. 5571330, pp. 307-309.

- GONZALEZ-BARBONE, V.; ANIDO-RIFON, L. Creating the first SCORM object. Computers and Education, 51 (4), 2008, pp. 1634-1647.

- Hotpotatoes. Disponível em http://hotpot.uvic.ca/. Acessado em 20 de maio de 2011

- IEEE. Draft Standard for Learning Object Metadata. IEEE 1484.12.1-2002

- MCKENNA, P. Reusable learning obstacles: Problems with the uptake of reusable learning objects in higher education. MCCSIS'08 - IADIS Multi Conference on Computer Science and Information Systems; Proceedings of e-Learning 2008, 2, pp. 219-222.

- MORALES, E.; GARCÍA, F.; RUIZ, Á.B. Research on learning-object management. ICEIS 2008 - Proceedings of the 10th International Conference on Enterprise Information Systems, AIDSS, 2008, pp. 559-562.

- MUSSOI, E. M. ; FLÔRES, M. L. P. ; BULEGON, Ana Marli ; TAROUCO, L. M. R. . GeoGebra and eXe Learning: applicability in the teaching of Physics and Mathematics. Journal of Systemics, Cybernetics and Informatics, v. 9, p. 61-66, 2011.

- OCHOA, X.; DUVAL, E. Measuring learning object reuse. Lecture Notes in Computer Science (including subseries Lecture Notes in Artificial Intelligence and Lecture Notes in Bioinformatics), 5192 LNCS, 2008, pp. 322-325.

- Open Content. Disponível em http://www.opencontent.org/ Acessado em 3 de maio de 2011

- SANZ-RODRIGUEZ, J.; DODERO, J.M.; SANCHEZ-ALONSO, S. Metrics-based evaluation of learning object reusability. Software Quality Journal, v.19, n.1, 2011. pp. 121-140.

- TAROUCO, Liane; DUTRA, Renato; ÁVILA, Barbara; GRANDO, Anita. SCORM e portabilidade: motivação e possibilidades. RENOTE - Revista Novas Tecnologias na Educação, v. 5, n. 1, 2007.

- WILEY, D. Impediments to Learning Object Reuse and Openness as a Potential Solution. Revista Brasileira de Informática na Educação, Volume 17, Número 3, 2009.

- WILEY, D. GIBSON, RECKER. A reformulation of the issue of learning object granularity and its implications for the design of learning objects. 2002 Disponível em http://reusability.org/granularity.pdf. Acessado em maio de 2011. 